\title{
What does Commercial Database Tell us About Medical Sciences?
}

\author{
Shinji Yokoyama
}

Nutritional Health Science Research Center, Chubu University, Kasugai, Japan

\section{See article vol. 25: 580-592}

Medical care in many industrialized counties became a part of essential infrastructure of societies either under public medical care system, like most of EU countries, Canada and Japan, or being covered by major private insurance companies, typically in The United States. Most of the data in such systems are digitalized in these days, including diagnosis, medical test results and transaction of expenses of every patient. It would therefore be easier than before to analyze these data to explore public health needs, examine cost-benefit balance and search marketing potential for drugs and medical devices if they are available and accessible. However, health care systems themselves may be reluctant to release these "raw" data freely for many reasons. Consequently, some private industries may start business to make those data commercially available based on contract with certain particular medical institutions. Medical Data Vision (MDV) Co. $\mathrm{Ltd}$. is one of those companies, who collects the data of the claims to Japanese public medical insurance systems from some 300 acute-care hospitals being uncoupled with patient identification and provides them on commercial basis.

Teramoto and his colleagues including investigators from Sanofi pharmaceutical company analyzed the data provided by MDV to examine the trend in treatment of the patients with elevated plasma LDL level in Japan ${ }^{1)}$. They intended to focus on familial hypercholesterolemia $(\mathrm{FH})$ to evaluate about how the patients are identified, diagnosed and treated in general practice. Among nearly 8 million patient data available, 77,415 patients were found 20 years-old or higher with LDL cholesterol measured enough to esti-

Address for correspondence: Shinji Yokoyama, Nutritional Health Science Research Center, Chubu University, Kasugai 487-8501, Japan

E-mail: syokoyam@isc.chubu.ac.jp

Received: December 7, 2017

Accepted for publication: December 14, 2017 mate the 2-year baseline, and 3,495 patients were identified as study population defined either as $\mathrm{FH}$ diagnosed (FH-D) to whom $\mathrm{FH}$ diagnosis was given by an attending physician (193) or as $\mathrm{FH}$-suspected (FH-S) whose LDL-cholesterol was $190 \mathrm{mg} / \mathrm{dL}$ or higher at least once in 2 years (3302). The authors analyzed the treatment given to these patients and concluded that $\mathrm{FH}$ is underdiagnosed and undertreated in Japan. The data are interesting and worthwhile publishing as we may see how high-LDL patients are "diagnosed" and treated in practice under the Japanese health care system. However, there are some issues the readers should be careful about when reading these data.

The authors discuss about the rate of diagnosis of $\mathrm{FH}$ and say it is underestimated in Japan referring to its prevalence in Japan estimated by Mabuchi et al. ${ }^{2)}$ based on the analysis of the patients in Hokuriku area. However, the authors in this reference themselves suggested that the estimated prevalence of $\mathrm{FH}, 1 / 208$, potentially includes founders effect in the area because one particular type of mutation accounts for as much as around $30 \%$ in this region while it is only $3 \%$ in Osaka area ${ }^{3)}$. Therefore, this number may be overestimation for general population. The prevalence of FH-D $1 / 401$ in the data analyzed by Teramoto et al. may rather represent general prevalence of $\mathrm{FH}$ among Japanese. If so, $\mathrm{FH}$ was diagnosed nearly $100 \%$ as far as people were measured for plasma cholesterol in acutecare hospitals in Japan. On the other hand, screening of FH-S does not seem based on the JAS FH guideline. Elevation of LDL-cholesterol is merely one of the criteria for $\mathrm{FH}$ in the guideline. Bring up 10-20 times more candidate subjects by screening is apparently overestimation. It may be inappropriate and mislead the audience to call them FH-S.

In addition, scientific credibility of the data in the claims to Japanese public medical insurance systems should also be questioned, especially the quality of diagnosis. The claims are submitted from medical facilities to ask reimbursement of their cost, which is strictly regulated by the national health insurance sys- 
tem with regard to relationship between diagnosis and procedures, prescription, treatment or clinical laboratory tests conducted. Therefore, the diagnoses in the claims have tendency to justify what have been done for the patients rather than medical accuracy. Their credibility is thus inevitably and substantially reduced by such a bias. The data of course show what is actually going on in medical practice in Japan and reflects "real world". However, there is no basic information to adjust these biases to increase their scientific credibility so that we must be very careful to interpret apparent findings based on these types of database.

We know that statistics of medical needs in public health inevitably depends on certain regulatory aspects of the system and can not avoid substantial dissociation from scientific reality. Therefore, we should not take these data as the ones similar to those collected in scientific programs such as epidemiological cohort studies or controlled clinical trials. Nevertheless, these data are still important and useful for us to make estimation of reality of medical practice as far as we realize the nature and limit of these databases.
What we should be afraid of is that using these types of database for "marketing research" of medical supplies may push the "market" further towards a wrong direction which may currently be already so.

\section{Conflict of Interest}

The author has no conflict of interest.

\section{References}

1) Teramoto T, Kai T, Ozaki A, Crawford B, Arai H, and Yamashita S. Treatment Patterns and Lipid Profile in Patients with Familial Hypercholesterolemia in Japan. J Atheroscler Thromb. 2018; 25: 580-592

2) Mabuchi H, Nohara A, Noguchi T, Kobayashi J, Kawashiri MA, Tada H, Nakanishi C, Mori M, Yamagishi M, Inazu A, Koizumi J: Molecular genetic epidemiology of homozygous familial hypercholesterolemia in the Hokuriku district of Japan, Atherosclerosis, 2011; 214: 404-407

3) Harada-Shiba M: Personal communication. 